\title{
NEUTRON EMISSION MEASUREMENTS OF PWR SPENT FUEL SEGMENTS AND PRELIMINARY VALIDATION OF DEPLETION CALCULATIONS
}

\author{
G. Perret ${ }^{1}$, D. Rochman ${ }^{1}$, A. Vasiliev $^{1}$ and H. Ferroukhi ${ }^{1}$ \\ ${ }^{1}$ Paul Scherrer Institut (PSI), Laboratory for Reactor Physics and Thermal-hydraulics (LRT) \\ Forschungsstrasse 111, 5232 Villigen PSI, Switzerland \\ gregory.perret@psi.ch,dimitri.rochman@psi.ch, alexander.vasiliev@psi.ch, \\ hakim.ferroukhi@psi.ch
}

\begin{abstract}
Assessing neutron emission of LWR spent fuel is necessary for the back-end of the fuel cycle, such as the dimensioning of transport and storage casks of spent fuel. Although core and depletion codes can calculate the isotopic composition of the discharged fuel and therefore infer its neutron source, accurate measured neutron emission values remain rare mainly because of the difficulty to prepare, handle and characterize spent fuel. Measured neutron emission values are, however, extremely relevant to code validation, as neutrons emitted by LWR spent fuel mainly originates from spontaneous fissions of minor actinides (e.g., ${ }^{242} \mathrm{Cm}$, ${ }^{244} \mathrm{Cm}$ and ${ }^{252} \mathrm{Cf}$ ) that are produced only after a large number of neutron captures in the reactor core. This paper reports on neutron emission measurements of selected LWR-PROTEUS spent fuel samples and their comparisons with a core and depletion calculation chains based on CASMO-5, SIMULATE-3 and the SNF codes. The measured LWR-PROTEUS samples are comprised of 11 samples irradiated in a Swiss PWR. The samples are $\mathrm{UO}_{2}$ or MOX and have discharge burn-ups ranging from 20 to $120 \mathrm{GWd} / \mathrm{t}$. We measured the $40-\mathrm{cm}$ long samples in a hot-cell of the Paul Scherrer Institut using a measurement station made of polyethylene and a $\mathrm{BF}_{3}$ detector. We repeated the measurements several times and in different conditions to ensure the accuracy and reproducibility of the results. We derived ratios of neutron rates emitted by the different samples and absolute neutron emission rates by comparison with a reference ${ }^{252} \mathrm{Cf}$ source, which we re-calibrated for this exercise. The experimental uncertainty $(1 \sigma)$ on the absolute neutron emission varies from $3 \%$ to $4 \%$. We compared a subset of the measured values to the calculation predictions and showed an agreement within less than $7 \%$ for all but one sample.
\end{abstract}

KEYWORDS: spent fuel, measurement, neutron emission, validation, LWR-PROTEUS

\section{INTRODUCTION}

The experimental characterization of spent fuel discharged from nuclear power plant is relevant for the back-end of the fuel cycle. In practice, results allow validating transport and depletion nuclear codes that are further used for assessing constraints related to the transport and storage of spent fuel (e.g., criticality safety, burn-up, decay heat, shielding issues in casks and storage pools) [1-4]. Nondestructive measurements (e.g. gamma ray and decay heat) are conducted at the fuel assembly level in the plant or in storage pools (e.g. [5-7]). At the pin level, nondestructive measurements - such as gamma ray profiles - 
are performed when inspecting rods in hot-cells. Destructive measurements relying on fuel samples extracted from fuel rods are also conducted for finer code validation, either on emitted gamma rays and fast neutrons [8] or on the isotopic composition of the samples (e.g. after dissolution) [9-11]. Measurements at the pin and sub-pin levels are rare because handling individual spent fuel rods generally requires highly regulated large infrastructures, such as hot-cells, and industrial partners providing the fuel in the first place.

Neutron emission measurements of spent fuel rods have an additional challenge, as the neutron-to-gammaray yield is very low for typical spent fuel $\left(>10^{-7}\right)$. This is usually solved by employing insensitive gammaray detectors like $\mathrm{BF}_{3}$ or ${ }^{3} \mathrm{He}$ detectors, which are thermal neutron detectors. In such setup, the fast neutrons emitted by the spent fuel need to be slow down before detection, which requires moderator and space. This comes at a cost in controlled environment like hot-cells because of: the safety regulations for material brought in hot-cell (e.g. criticality and fire hazard), the need to design specific measurement station operable by tele manipulators, and the need to decontaminate the equipment after the measurement. Neutron emission measurements are, however, relevant for code validation as neutrons emitted by LWR spent fuel mainly originates from spontaneous fissions of minor actinides (e.g., ${ }^{242} \mathrm{Cm},{ }^{244} \mathrm{Cm}$ and ${ }^{252} \mathrm{Cf}$ ) that are produced only after a large number of neutron captures in the reactor core and are a challenge to predict accurately.

The Hot-Laboratory (AHL) at the Paul Scherrer Institut (PSI) operates a set of hot-cell and routinely examines spent fuel provided by the Swiss nuclear power plants. In the context of the LWR-PROTEUS program, which was a collaboration between PSI and the Swiss Nuclear Power Plants (swissnuclear), a set of 13 spent fuel segments were cut at AHL from $\mathrm{UO}_{2}$ and MOX fuel rods irradiated in PWR and BWR. These segments are $40-\mathrm{cm}$ long and have flat burn-up profiles (i.e. constant axial gamma emission distributions). Their burn-up ranges from 20 to $120 \mathrm{GWd}$ /t. In addition to the LWR-PROTEUS segments, portions of the original spent fuel rod adjacent to the segments were cut and examined destructively, yielding the isotopic content of the spent fuel segments [12].

The LWR-PROTEUS segments are an ideal set for validation because they are very well characterized experimentally, span a large set of conditions found in reactors and because the irradiation history of the original fuel rods is modeled in details at the Laboratory for Reactor Physics and Thermal-hydraulic (LRT) of PSI. In the past, these samples were used to validate the predictions of deterministic and Monte-Carlo depletion calculations in terms of isotopic composition and burn-up predictions [13, 14], and develop methods to propagate the uncertainties due to nuclear data (e.g. cross-sections and fission yields) in these calculations [15]. In the context of burn-up credit, the reactivity worth of the samples compared to that of fresh fuel was also measured and used for code validation [16]. Again, this reactivity measurement served as a test bed for benchmarking uncertainty propagation methods for deterministic and stochastic codes [1719]. Finally, the neutron emissions of the samples were already measured by the source multiplication technique in the zero-power reactor PROTEUS in subcritical conditions and correlated to their reactivity worths [20].

In this paper and in the context of the CASCADES collaborative project between PSI and swissnuclear, we measured anew, and with a different technique, the neutron emission of the PWR segments issued from the LWR-PROTEUS set. The paper focus on the description of the measurement and the experimental results. The validation effort is underway and is reported for a subset of the measured samples. Section 2 presents the material used for the measurement. Section 3 presents the measurement campaign and the results together with the method of analysis. Finally, Section 4 presents the code chain used for the validation and the associated results.

\section{MATERIALS}


We measured the 11 LWR-PROTEUS spent fuel samples issued from PWR fuel rods in hot cell 5 at AHL. The samples are $40-\mathrm{cm}$ long and overcladded in Zircaloy. Their main characteristics are recalled in Table I.

Table I.: Measured LWR-PROTEUS segments characteristics.

\begin{tabular}{|c|c|c|c|c|c|}
\hline Fuel Type & Id. & $\begin{array}{c}\text { Cycles } \\
\text { irradiated }\end{array}$ & $\begin{array}{c}\text { Number of } \\
\text { relocation }\end{array}$ & $\begin{array}{c}\text { Burnup } \\
{[\mathrm{GWd} / \mathrm{t}]}\end{array}$ & $\begin{array}{c}\text { Shutdown } \\
\text { date }\end{array}$ \\
\hline \multirow{7}{*}{$\mathrm{UO}_{2}$} & U1 & 2 & - & $\sim 40$ & 1996/06 \\
\hline & $\mathrm{U} 2$ & 3 & - & $\sim 50$ & 1995/06 \\
\hline & U3 & 5 & 1 & $\sim 70$ & 1995/06 \\
\hline & U4 & 5 & 1 & $\sim 70$ & 1995/06 \\
\hline & U5 & 7 & 3 & $\sim 90$ & 1995/06 \\
\hline & U6 & 7 & 3 & $\sim 90$ & $1995 / 06$ \\
\hline & U7 & 10 & 4 & $\sim 120$ & $2001 / 07$ \\
\hline \multirow{4}{*}{ MOX } & M1 & 1 & - & $\sim 20$ & $1998 / 06$ \\
\hline & M2 & 2 & - & $\sim 40$ & $1999 / 06$ \\
\hline & M3 & 3 & - & $\sim 60$ & $2000 / 07$ \\
\hline & M4 & 4 & - & $\sim 70$ & $2001 / 07$ \\
\hline
\end{tabular}

The measurement station is made of polyethylene with a density of $0.95 \mathrm{~g} / \mathrm{cc}$, measures $40 \times 60 \times 30 \mathrm{~cm}^{3}$ and weights $72 \mathrm{~kg}$. The station contains a channel to hold the spent fuel samples or the reference ${ }^{252} \mathrm{Cf}$ source and four separate holes to accommodate a $\mathrm{BF}_{3}$ detector. The holes are drilled at different distances to the channel containing the spent fuel sample/source and are numbered from 1 to 4 starting from the nearest hole. While we inserted the detector in one hole, we inserted polyethylene plugs in the others to keep the geometry simple. The different holes allow measuring with different absolute detection efficiency and accommodating the neutron emission rate of the spent fuel samples. Figure 1 (left) shows top and side views of the measurement station, with the source or detector holders.

The $\mathrm{BF}_{3}$ detector used in the measurement campaign ( $\mathrm{MN}-1$ type from Centronics) has a nominal active length of $127 \mathrm{~mm}$ and a total length of $184 \mathrm{~mm}$. The nominal active diameter is $6.3 / 6.4 \mathrm{~mm}$ and that of the casing is $7.5 \mathrm{~mm}$. The $\mathrm{BF}_{3}$ gas is enriched in ${ }^{10} \mathrm{~B}$ and is at a pressure of $20 \mathrm{~cm} \mathrm{Hg}$. The detector is operated at the nominal high voltage of $1100 \mathrm{~V}$ and has an intrinsic sensitivity of $\sim 5 \times 10^{-2} \mathrm{cps} / \mathrm{nv}$. The detector is housed in a piece of Teflon and a Peraluman holder (see Figure 1 - right). The Teflon piece comprises two cylinders $\left(\mathrm{H}=12 \mathrm{~cm}, \varnothing_{\mathrm{ext}}=4 \mathrm{~cm}\right.$ and $\left.\mathrm{H}=5 \mathrm{~cm}, \varnothing_{\mathrm{ext}}=3.3 \mathrm{~cm}\right)$ with an inner hole allowing to slide the $\mathrm{BF}_{3}$ detector. The nominal position for the center of the detector (along its length) is $5.3 \mathrm{~cm}$ deeper in the hole than the source/fuel sample center. The $\mathrm{BF}_{3}$ detector has a 5-m long cable and we operated it with a single channel analyzer and a scaler making the electronics dead time negligible for the measured count rates.

$\mathrm{A}^{252} \mathrm{Cf}$ source is used to derive the absolute neutron emission rates for the LWR-PROTEUS samples. The source active part is a cylinder with a height of $0.5 \mathrm{~cm}$ and a diameter of $0.5 \mathrm{~cm}$, which is centered in a stainless steel cylinder with an outer diameter of $0.78 \mathrm{~cm}$ and a height of $1 \mathrm{~cm}$. The source is mounted on an extension piece (see Figure 1 - right), which is a 14-cm long cylinder of aluminum with an external diameter of $0.78 \mathrm{~cm}$. The source and its extension fit in the source holder made of Peraluman that insures its correct positioning and an easy handling in the hot cell (see Figure 1 - right). When fully inserted into the measurement station, the source has its center aligned with that of the spent fuel samples. The source holder features four marks that allow a reproducible axial displacement of the source from $5 \mathrm{~cm}$ to $20 \mathrm{~cm}$. This displacement is used for the axial geometrical characterization of the detection process (see 
Section 3.1). Because the source was manufactured in April 1988, the impurity in ${ }^{250} \mathrm{Cf}$ and the spontaneous fissions from ${ }^{248} \mathrm{Cm}$ ( $\alpha$-decay product of ${ }^{252} \mathrm{Cf}$ ) represent a non-negligible part of the total source intensity - mainly as ${ }^{252} \mathrm{Cf}$ has decayed faster than ${ }^{250} \mathrm{Cf}$. Because we do not have a certificate for the ${ }^{250} \mathrm{Cf}$ impurities, we measured anew the source intensity by cross-validation against another well-known ${ }^{252} \mathrm{Cf}$ source within two weeks of the measurement campaign. At the date of the measurement campaign at AHL, the source intensity and its $1 \sigma$ uncertainty were $(189.4 \pm 5.5) \times 10^{3} \mathrm{n} / \mathrm{s}(2.9 \%)$ emitted in $4 \pi[21]$.
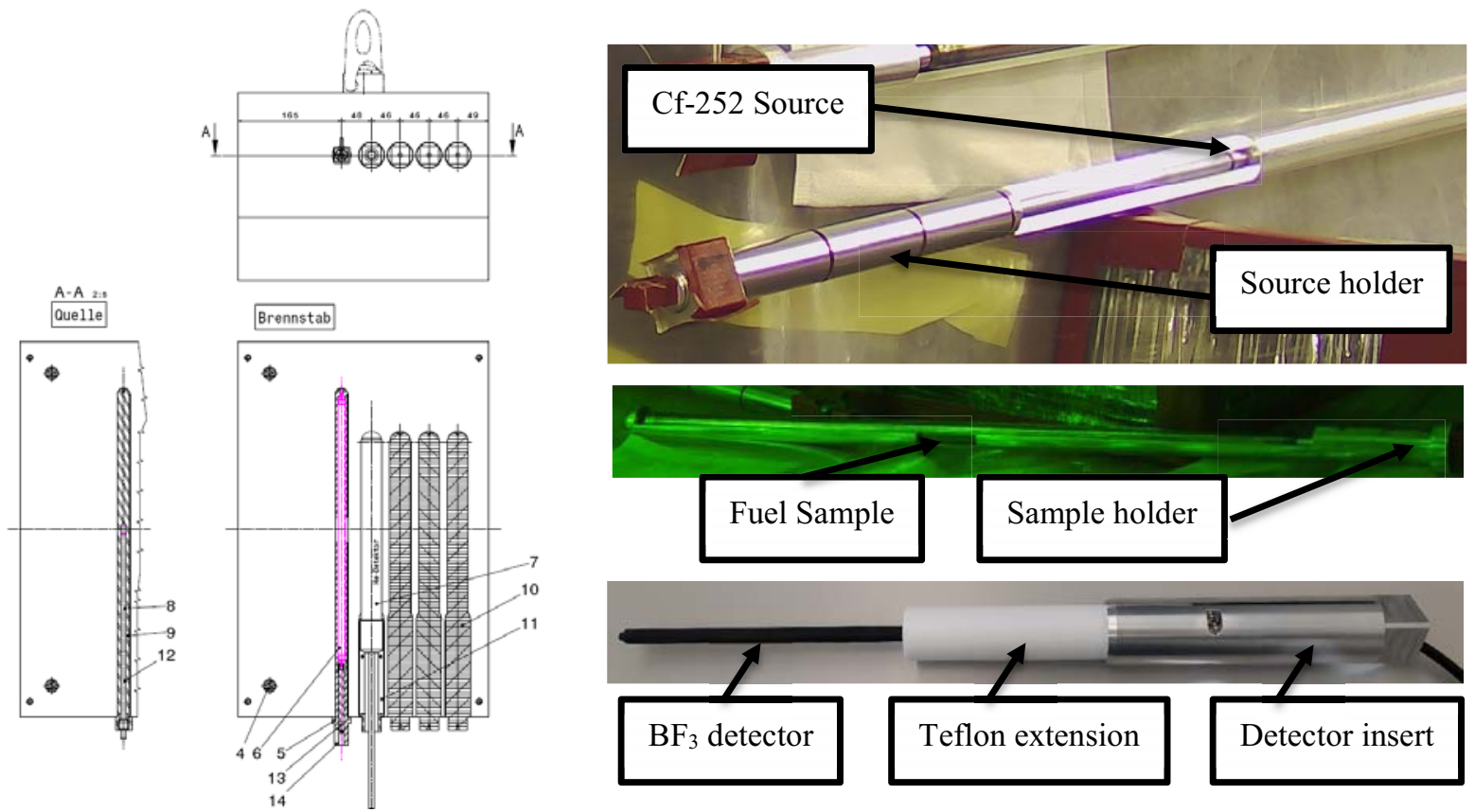

Figure 1. Measurement station top and side view (left); pictures of source holder, sample holder and $\mathrm{BF}_{3}$ detector (right).

\section{EXPERIMENTAL RESULTS}

We conducted the measurements from April 30 to May 3 2018. We measured each spent fuel sample several times in the same position to check the reproducibility of the measurement and improve the statistics. We also measured the samples with the detector inserted in different holes (1 to 4) depending on the neutron emission of the sample. The $1 \sigma$ uncertainty on the measured counts after aggregation vary between $0.3 \%$ and $0.9 \%$. We used sample $\mathrm{U} 3-\mathrm{UO}_{2}$ with nominal burnup value of $\sim 70 \mathrm{GWd} / \mathrm{t}$ - to normalize the neutron emission rates and yield the relative neutron emission rates. We therefore measured sample U3 with the detector inserted in every holes. The $1 \sigma$ uncertainty on the relative counts remains lower than $1.1 \%$. Finally, to yield the absolute neutron emission rates of the spent fuel sample we measured the ${ }^{252} \mathrm{Cf}$ source with the detector inserted in all four holes (with the source holder fully inserted in the channel).

\subsection{Relative Neutron Emission Rates}

The relative neutron emission rates is the ratio of the count rates as the samples have the same geometry (i.e., no solid angle corrections is expected) and the samples have large cooling times (i.e., more than $99 \%$ of the neutron emissions is due to spontaneous fission of ${ }^{244} \mathrm{Cm}$ and no spectral correction is required). The only exception is sample U7, which has a very high burn-up - $120 \mathrm{GWd} / \mathrm{t}$ - and for which the number of neutrons emitted by spontaneous fissions of ${ }^{252} \mathrm{Cf}$ may not completely negligible. However, we estimated the ${ }^{252} \mathrm{Cf}$-to- ${ }^{244} \mathrm{Cm}$ neutron emission rate for this sample to be $5.7 \%$ at the measurement date [22]. Because 
the latter value is small and because the spontaneous fission emission spectra of ${ }^{252} \mathrm{Cf}$ and ${ }^{244} \mathrm{Cm}$ are similar, we neglect any spectral correction at this stage.

Following the above considerations, the relative count rates for each sample " $x$ " obtained with the detector located in any holes "h" should be similar. A weighted mean, as defined by Eq. (1), should give a reliable estimate for the relative neutron rate and its uncertainty.

$$
\langle x\rangle \pm \sigma_{\langle x\rangle}=\frac{\sum_{h} w_{h} x_{h}}{\sum_{h} w_{h}} \pm \sqrt{\frac{1}{\sum_{h} w_{h}}}, \quad w_{h}=\frac{1}{\sigma_{h}^{2}}
$$

In Eq. (1), $\sigma_{\langle x\rangle}$ is the standard error of the mean $\langle\mathrm{x}\rangle$ and $\sigma_{\mathrm{h}}$ is the standard deviation on the relative count rate $\mathrm{x}_{\mathrm{h}}$. However, to check that the relative count rates in actually the same in each hole, we also compute the empirical mean $\bar{x}=1 / N \sum x_{h}$ and its standard error $\sigma_{\bar{x}}=s / \sqrt{N}$, which is calculated from the standard deviation $s=\sqrt{1 /(N-1) \sum\left(x_{h}-\bar{x}\right)^{2}}$. Results for the measurement with the detector in Hole 4 generally differ from the others. This translates for the concerned samples in $\sigma_{\bar{x}}$ being larger than $\sigma_{\langle\mathbf{x}\rangle}$. Because we cannot explain the bias for measurement in Hole 4, we chose to keep the estimate $\langle\mathrm{x}\rangle$ for the mean value. However, to be conservative, we consider the standard error to be $\max \left(\sigma_{\langle x\rangle}, \sigma_{\bar{x}}\right)$. Mean and $1 \sigma$ standard error results are listed in Table II of Section 3.2 with that for the absolute neutron emissions rates. Samples are ordered by increasing emissions.

\subsection{Absolute Neutron Emission Rates}

Absolute neutron emission rates are derived from the ratio of the count rates obtained from the spent fuel samples and from the ${ }^{252} \mathrm{Cf}$ source, as well as from the intensity of the ${ }^{252} \mathrm{Cf}$ source. The differences in sample/source geometry (40-cm vs. 1 -cm long) and neutron emission spectra $\left({ }^{244} \mathrm{Cm}\right.$ vs. $\left.{ }^{252} \mathrm{Cf}\right)$ are corrected by a combination of experiments and Monte-Carlo modeling.

We characterized the main geometrical effect experimentally by moving the quasi-punctual ${ }^{252} \mathrm{Cf}$ source from the center of the measurement station to the side by steps of 5-cm using the markings on the source holder (see Figure 1 - right). We repeated the exercise for the detector being positioned in each hole. The resulting axial profiles were then integrated from $0 \mathrm{~cm}$ to $20 \mathrm{~cm}$ and normalized per centimeter to obtain a correction factor representative of the geometrical correction between a punctual source and a $40-\mathrm{cm}$ long sample. These factors are, however, only approximate geometrical corrections as they do not account for the dissymmetry of the measurement station (namely that the holes in which the sample/source is inserted is closed on one side - see Figure 1 - right). We, however, used these axial profiles to calibrate MonteCarlo simulations that could account for the dissymmetry and the difference in the neutron emission spectra.

The Monte-Carlo simulations were conducted with MCNP6.1.1 [23] as shown in Figure 2 for selected configurations. We modeled the measurement station and the whole hot-cell according to specifications with some simplifications (e.g., screws in stainless steel not modeled as they are located away from the detector and the source; the geometry of the source and detector holders is simplified as the holders are made of Peraluman). Given the specifications of the measurement station, sensitivity studies confirmed that the hot cell structure do not influence the measurement. We used cross-sections from JEFF-3.1.1 [24] and run up to $5 \times 10^{8}$ neutrons. In each modeled configuration, the tallied ${ }^{10} \mathrm{~B}(\mathrm{n}, \alpha)$ reactions in the $\mathrm{BF}_{3}$ detector had a relative $1 \sigma$ uncertainty lower than $1 \%$.

Before deriving the spatial and spectral correction factors by comparing calculations with the source and the spent fuel samples, we calibrated the model by reproducing the axial profile measured experimentally with the ${ }^{252} \mathrm{Cf}$ source. We changed several parameters of the simulation (e.g., uncertainty in material density, location of holes, location of detector, etc.) and showed that the location of the sensitive volume in the $\mathrm{BF}_{3}$ 
detector is the only factor significant enough to allow calibrating the simulations. Once calibrated, we could reproduce the axial profile and yield the experimental correction factors within $\pm 2.5 \%$. The calibrated simulations were then used to correct for the total geometrical effect (length and diameter of the source) and the spectral differences. The correction factors with their $1 \sigma$ uncertainties originating from the statistical uncertainty of the Monte-Carlo calculations are for Holes 1 to $4: 0.461(0.3 \%), 0.485(0.5 \%), 0.520(0.9 \%)$ and $0.565(1.3 \%)$, respectively.

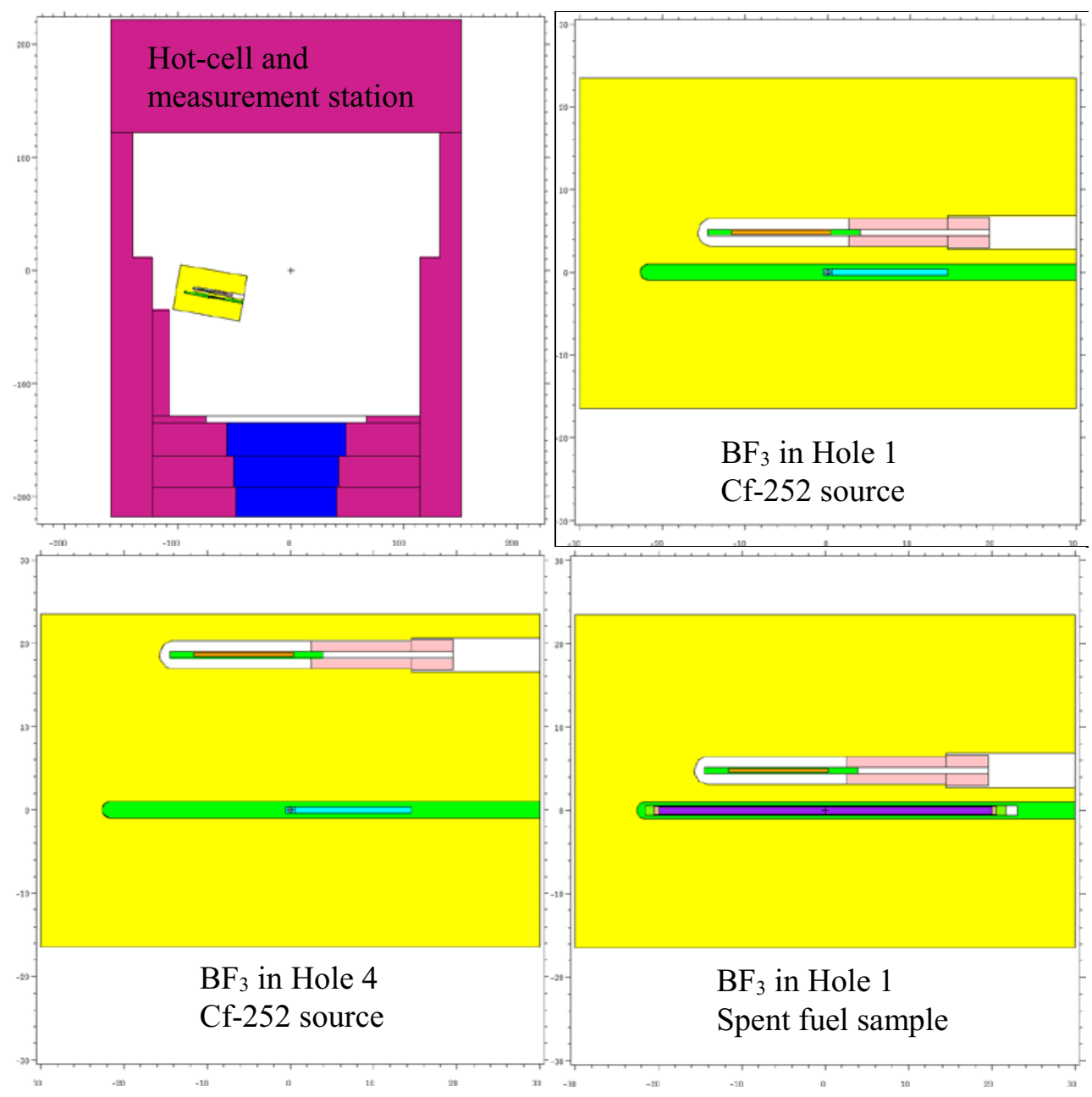

Figure 2. Horizontal cutaway views of the MCNP model of selected measurement set-ups distances in $\mathbf{c m}$.

We estimate the absolute neutron emission rate per neutron emitted by the Cf- 252 source as $n_{h}$ for each spent fuel samples with the detector inserted in Hole $h$ as:

$$
n_{h}=\frac{y_{h}}{s_{h}} \frac{1}{f_{h}}
$$

where $y_{h}$ and $s_{h}$ are the count rates of the sample and of the ${ }^{252} \mathrm{Cf}$ source with the detector inserted in Hole $h$ and $f_{h}$ is the correction factor that accounts for geometrical and spectral differences between the source and the detector. For each sample, we averaged the results on the measurements performed with the detector located in different holes in the same way than for the relative emission rates. The results from 
measurements in Hole 4 were again slightly different from the others, and for that reason, we considered the weighted mean (Eq. (1)) to be the best estimate and took $\max \left(\sigma_{\langle n\rangle}, \sigma_{\bar{n}}\right)$ as the standard error on that estimate. The uncertainty $\sigma_{\langle n\rangle}$ already accounts for the uncertainty on the count rates and the correction factors. The absolute neutron emission rate of each sample is: $\bar{n}$, where $S$ is the ${ }^{252} \mathrm{Cf}-252$ source intensity. Results are listed in Table II with their $1 \sigma$ uncertainty, which accounts for the $2.9 \%$ uncertainty on $S$ (see Section 2).

Table II.: Measured relative and absolute neutron emission rates with their $1 \sigma$ uncertainties.

\begin{tabular}{|c|c|c|c|}
\hline $\begin{array}{c}\text { Probe } \\
\text { ID }\end{array}$ & $\begin{array}{c}\text { Burnup } \\
{[\mathrm{GWd} / \mathrm{t}]}\end{array}$ & $\begin{array}{c}\text { Relative } \\
{[\text { a.u.] }}\end{array}$ & $\begin{array}{c}\text { Absolute } \\
{\left[\mathrm{x} 10^{3} \mathrm{n} / \mathrm{s}\right]}\end{array}$ \\
\hline U1 & $\sim 40$ & $0.05779 \pm 0.00052(0.9 \%)$ & $40.7 \pm 1.2(3.0 \%)$ \\
\hline U2 & $\sim 50$ & $0.3140 \pm 0.0019(0.6 \%)$ & $221 \pm 7(3.0 \%)$ \\
\hline M1 & $\sim 20$ & $0.5005 \pm 0.0029(0.6 \%)$ & $352 \pm 10(3.0 \%)$ \\
\hline U3 & $\sim 70$ & 1.000 & $700 \pm 24(3.5 \%)$ \\
\hline U4 & $\sim 70$ & $1.0814 \pm 0.0045(0.4 \%)$ & $758 \pm 22(3.0 \%)$ \\
\hline M2 & $\sim 40$ & $1.645 \pm 0.019(1.1 \%)$ & $1151 \pm 34(3.0 \%)$ \\
\hline U5 & $\sim 90$ & $1.895 \pm 0.012(0.6 \%)$ & $1328 \pm 40(3.0 \%)$ \\
\hline U6 & $\sim 90$ & $2.022 \pm 0.014(0.7 \%)$ & $1407 \pm 54(3.8 \%)$ \\
\hline M3 & $\sim 60$ & $3.035 \pm 0.034(1.1 \%)$ & $2117 \pm 88(4.2 \%)$ \\
\hline M4 & $\sim 70$ & $3.851 \pm 0.057(1.5 \%)$ & $2702 \pm 117(4.3 \%)$ \\
\hline U7 & $\sim 120$ & $5.663 \pm 0.068(1.2 \%)$ & $3953 \pm 165(4.2 \%)$ \\
\hline
\end{tabular}

\section{CODE VALIDATION}

\subsection{Code Chain Description}

We calculated the absolute neutron emission rates based on validated models used for core cycle licensing analysis with the CASMO-5 and SIMULATE-3 codes [25, 26]. The SNF-1.6 code [27] allowed us to extract the assembly and sample characteristics.

More specifically, validated models for CASMO-5 and SIMULATE-3 are available for all the irradiation cycles of the nuclear power plant that hosted the LWR-PROTEUS samples. Such models (made of the descriptions of assemblies and cycles) include all necessary information: assemblies and core geometry, enrichments, fuel patterns, etc. We routinely compare their predictions with boron letdown and in-core fission rate measurements (see e.g. [28]). The SNF-1.6 code then extracts selected assembly, pin and node data. The code uses the assembly geometry and fuel details data from CASMO-5 and the irradiation histories and pin power reconstruction results from SIMULATE-3. Whereas we did not validate SNF and its inputs in LRT, Studsvik validated the code with various decay heat measurements. The code can calculate different quantities such as decay heat, number of emitted neutrons and gammas, or isotopic concentrations, all as a function of irradiation and cooling time. In the present work, we extract the neutron emission from the SNF output for the nodes containing the $10-\mathrm{mm}$ long segments destructively measured at AHL to determine the isotopic composition of the LWR-PROTEUS samples, which can be compared with the measured values presented in Section 3. 


\subsection{Validation Results and Discussion}

For this comparison, we limit ourselves to the samples that were not relocated during the irradiation in the nuclear power plant. In addition, because the calculations were originally performed for a reference date in November 2003 we corrected the experimental values for the build-up of ${ }^{244} \mathrm{Cm}$ using the $\sim 18.1$-year halflife of JEFF-3.1.1. This is legitimate as more than $99 \%$ of the neutron emission for all considered samples comes from ${ }^{244} \mathrm{Cm}$ in November 2003 and May 2018. Calculation-to-experimental ratios (C/E) are listed in Table III, with the $1 \sigma$ uncertainty and the associated confidence interval at $95 \%$. At this stage, we consider only the experimental uncertainty and not that on the calculations coming for example from the nuclear data uncertainties.

Table III.: Comparison of predicted and measured absolute neutron emission rates.

\begin{tabular}{|c|c|c|c|}
\hline $\begin{array}{c}\text { Probe } \\
\text { ID }\end{array}$ & $\begin{array}{c}\text { Burnup } \\
{[\mathrm{GWd} / \mathrm{t}]}\end{array}$ & $\mathrm{C} / \mathrm{E}$ & Conf. Int. (95\%) \\
\hline U1 & $\sim 40$ & $0.743 \pm 0.023(3.0 \%)$ & {$[0.699,0.788]$} \\
\hline U2 & $\sim 50$ & $1.031 \pm 0.031(3.0 \%)$ & {$[0.971,1.091]$} \\
\hline M1 & $\sim 20$ & $0.916 \pm 0.027(3.0 \%)$ & {$[0.863,0.969]$} \\
\hline M2 & $\sim 40$ & $0.997 \pm 0.030(3.0 \%)$ & {$[0.938,1.055]$} \\
\hline M3 & $\sim 60$ & $1.064 \pm 0.044(4.2 \%)$ & {$[0.977,1.151]$} \\
\hline M4 & $\sim 70$ & $1.021 \pm 0.044(4.3 \%)$ & {$[0.935,1.107]$} \\
\hline
\end{tabular}

Measurements and predictions of the absolute neutron emissions agree (i.e. within the confidence interval at $95 \%$ ) for all samples except the low burn-up samples U1 and M1. The agreement is, however, within $10 \%$ for all samples but U1. The $25 \%$ disagreement for sample U1 is probably due to the axial locations of the measured sample and the 10-mm long adjacent sample for which the calculations were performed. In that region, the axial neutron flux and resulting burn-up profile is not completely flat. This is confirmed by the ${ }^{137} \mathrm{Cs}$ gamma scanning of the original rod (before extraction of the samples) that shows a decrease of the gamma emissions (and corresponding burn-up) from the LWR-PROTEUS sample to the 10-mm long adjacent sample. This decrease is consistent with the under prediction of the calculation observed in Table III.

\section{CONCLUSIONS}

We measured the relative and absolute neutron emission rates of the 11 PWR spent fuel samples manufactured during the LWR-PROTEUS Phase II program. The $\mathrm{UO}_{2}$ and $\mathrm{MOX}$ spent fuel samples have burnups ranging from $20 \mathrm{GWd} / \mathrm{t}$ to $120 \mathrm{GWd} / \mathrm{t}$. We performed the measurements at the Hot Laboratory of PSI using a measurement station made of Polyethylene blocks to slow down the fast neutrons emitted by

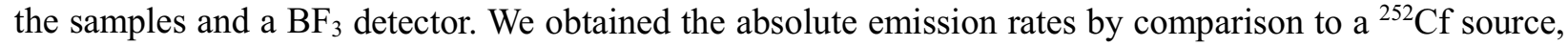
whose intensity we calibrated at the same time than the measurements. We compared absolute emission rates to preliminary predictions by the CASMO/SIMULATE/SNF code chains.

The measured relative neutron emission rates do not require geometrical or spectral corrections and should therefore not exhibit any significant bias. The uncertainty on the samples vary from $0.4 \%$ to $1.5 \%(1 \sigma)$. Yielding the absolute neutron emission rates requires a geometrical correction (mainly due to the 1-cm long source vs. the $40-\mathrm{cm}$ long spent fuel segment) and a slight spectral correction $\left({ }^{252} \mathrm{Cf}\right.$ vs. ${ }^{244} \mathrm{Cm}$ spontaneous fission emission spectra). The corrections were obtained from an MCNP model that was calibrated against a partial measurement of the axial profile obtained by moving the ${ }^{252} \mathrm{Cf}$ source along the length of the channel of the measurement station. In the worst case, this correction factors calibration could introduce a 
small bias of $2.5 \%$. Biases on the source intensity (as the source is old and the ${ }^{250} \mathrm{Cf}$ content is not known see Section 2) were taken into account thanks to the source re-calibration. The uncertainty on the measured absolute neutron emission rates accounts for the statistical uncertainty on the measurement, the stochastic uncertainty of the correction factor obtained with MCNP and that on the source intensity. The latter is estimated at $2.9 \%(1 \sigma)$ and is dominant. The total uncertainty on the measured absolute neutron emission rates varies from $3.0 \%$ to $4.3 \%(1 \sigma)$.

Comparisons of the measured absolute neutron emission rates with preliminary predictions by the CASMO/SIMULATE/SNF code chain showed agreement within $10 \%$ for most samples. The only exception is sample U1, for which predictions are $\sim 25 \%$ lower than experiments. At the time of the writing, we did not calculate any uncertainty on the predicted neutron emissions. Despite that, four of the six predictions agree within $2 \sigma$ with the experimental values. The validation reported here is only preliminary and serves to illustrate the good agreement with the experiments. We will extend the validation in future work by assessing the neutron outputs of the 11 PWR samples and deriving uncertainty on their values.

\section{ACKNOWLEDGMENTS}

The LWR-PROTEUS spent fuel samples measured in this work were manufactured during the LWRPROTEUS program, which was conducted jointly by Paul Scherrer Institut (PSI) and the Swiss Nuclear Power Plants (swissnuclear), with specific contributions from Kernkraftwerk Gösgen (KKG) for Phase II. The work reported in this paper is part of the CASCADES research project conducted jointly by PSI and swissnuclear. We would like to particularly thank Hans Leu and Andreas Spahr for helping performing the measurements at the Hot Laboratory; D. Kuster, C. Gerber and M. Streit for helping preparing the measurements; and U. Sigrist for helping designing the measurement station.

\section{REFERENCES}

1. I. C. Gauld and J. C. Ryman, "Nuclide Importance to Criticality Safety, Decay Heating, and Source Terms Related to Transport and Interim Storage of High-Burnup LWR Fuel - NUREG/CR-6700," Oak Ridge, TN 37831-6370, 2000.

2. I. C. Gauld and C. V. Parks, "Review of Technical Issues Related to Predicting Isotopic Compositions and Source Terms for High-Burnup LWR Fuel - NUREG/CR-6701," Oak Ridge, TN 37831-6370, 2000.

3. B. B. Bevard, J. C. Wagner, C. V. Parks, and M. Aissa, "Review of Information for Spent Nuclear Fuel Burnup Confirmation - NUREG/CR-6998," 2009.

4. J. C. Wagner, M. D. DeHart, and C. V. Parks, "Recommendations for Addressing Axial Burnup in PWR Burnup Credit Analyses - NUREG/CR-6801," 2003.

5. K. Abbas, G. Nicolaou, and L. Koch, "In situ gamma spectroscopy of spent nuclear fuel using a CdTe detector," Nucl. Instruments Methods Phys. Res. A, 383(2-3), pp. 601-604 (1996).

6. J. Vogt et al., "Measurements of decay heat and gamma-ray intensity of spent LWR fuel assemblies," IAEA-SM-352/40.

7. M. V. Mora, "Nondestructive burnup measurements by gamma-ray spectroscopy on spent fuel elements of the RP-10 research reactor," Progress in Nuclear Energy, 53, pp. 344-353 (2011).

8. A. Sasahara et al., "Neutron and Gamma Ray Source Evaluation of LWR High Burn-up $\mathrm{UO}_{2}$ and MOX Spent Fuels," J. Nucl. Sci. Technol., 41 (4), pp. 448-456 (2004).

9. A. Sasahara et al., "Isotopic Analysis of Actinides and Fission Products in LWR High-Brunup UO2 Spent Fuels and its Comparison with Nuclide Composition Calculated Using JENDL, ENDF/B, JEF, and JEFF," J. Nucl. Sci. Technol., 45 (4), pp. 313-327, (2008).

10. A. Sasahara et al., "Chemical Isotopic Analysis of Fission Products in PWR-MOX Spent Fuels and Computational Evaluation Using JENDL, ENDF/B, JEF, and JEFF," J. Nucl. Sci. Technol., 45 (5), pp. 390-401, (2008). 
11. H. U. Zwicky et al., "Nuclide analysis in high burnup fuel samples irradiated in Vandellós 2," J. Nucl. Mater., vol. 402, no. 1, pp. 60-73, Jul. 2010.

12. P. Grimm, I. Günther-Leopold and H.D. Berger, "Burnup calculations and chemical analysis of irradiated fuel samples studied in LWR-PROTEUS Phase II," Proceedings of Physics of reactors 2016: (PHYSOR 2006), Vancouver, Canada, Sept. 10-14 (2006).

13. P. Grimm, G. Perret and H. Ferroukhi, "CASMO-4E AND CASMO-5 analysis of the isotopic compositions of the LWR-PROTEUS phase II burnt PWR $\mathrm{UO}_{2}$ fuel samples", Proceedings of Physics of reactors 2014: the role of reactor physics toward a sustainable future (PHYSOR 2014), Kyoto, Japan, Oct. 3, A (2014).

14. M. Pecchia et al., "Validation of Monte Carlo based burnup codes against LWR-PROTEUS Phase-II experimental data," Ann. Nucl. Energy, 97, pp. 153-164 (2016).

15. O. Leray et al., "Nuclear data uncertainty propagation on spent fuel nuclide compositions," Ann. Nucl. Energy, 94, pp. 603-611 (2016).

16. P. Grimm et al., "Analysis of Reactivity Worths of Highly-Burnt PWR Fuel Samples Measured in LWRPROTEUS Phase II," Proceedings of Physics of reactors 2008: (PHYSOR 2008), Interlaken, Switzerland, Sept. 14-19 (2008).

17. M. Hursin et al., "Verification of the new implementations in SHARK-X for reactivity coefficients and relative reactivity worth UQ", Proceedings of Physics of reactors 2016: Unifying Theory and Experiments in the 21st century (PHYSOR 2016), May 1-5, 1 (2016).

18. P. Grimm et al., "Analysis of reactivity worths of burnt PWR fuel samples measured in LWRPROTEUS Phase II using a CASMO-5 reflected-assembly model," Prog. Nucl. Energy, 101 (2017).

19. J. Park et al., "Uncertainty quantification of LWR-PROTEUS Phase II experiments using CASMO-5," Ann. Nucl. Energy, 131(9), pp. 9-22 (2019).

20. M.F. Murphy et al., "Reactivity and neutron emission measurements of highly burnt PWR fuel rod samples," Ann. Nucl. Energy, 33(9), pp. 760-765 (2006).

21. G. Perret and U. Strauch, "Measurement of Cf-252 Source \# 1154 Intensity," Paul Scherrer Institute, AN-41-18-09-V.0 (2018).

22. G. Perret and D. Rochman, "Neutron emission measurement of the PWR LWR-PROTEUS Phase II spent fuel samples at AHL," Paul Scherrer Institute, TM-41-18-17 V.0 (2018).

23. J.T. Goorley, "MCNP6.1.1-Beta Release Notes," Los Alamos National Laboratory, LA-UR-14-24680 (2014).

24. A. Santamarina et al., "The JEFF-3.1.1 Nuclear Data Library," Data Bank, JEFF Report 22, NEA No. 6807, OECD-NEA (2009).

25. J. Rhodes et al., "CASMO-5 User's Manual Rev. 5," Studsvik, SSP-07/431 Rev. 5, 2012.

26. A.S. DiGiovine et al., "SIMULATE-3 User's Manual," Studsvik, Studsvik/SOA-95/15 (1995).

27. T. Simeonov et al., "SNF User's Manual," Stusvik, SSP-11/328 Rev.3 (2011).

28. H. Ferroukhi, "ENSI On-Call 2018 - Core Licensing Analyses for KKG Cycle 40," Paul Scherrer Institute, TM-41-18-14-V.0. (2018). 